\title{
Sleepy and hostile: The effects of REM sleep deprivation on shock-elicited aggression
}

\author{
MARY NELL MOLLENHOUR, JAMES W. VOORHEES, and STEPHEN F. DAVIS \\ Austin Peay State University, Clarksville, Tennessee 37040
}

\begin{abstract}
Two studies were conducted to assess the effects of rapid eye movement (REM) sleep deprivation on shock-elicited aggression. REM deprivation periods of $0,24,48$, and $72 \mathrm{~h}$ were used in the first study, while 48-, 72-, 96-, and 120-h periods were used in the second study. Both studies indicated that increases in REM deprivation (up to $96 \mathrm{~h}$ ) resulted in increases in the number of aggressive responses. A decrease was shown by the 120 -h group. A drive-energization or motivational-effects model is suggested.
\end{abstract}

Subsequent to the publication of findings by Ulrich and Azrin (1962), pain-elicited aggression has become a topic of considerable interest. Attempting to elucidate a phenomenon previously described by O'Kelly and Steckle (1939), Ulrich and Azrin (1962) determined that shock-induced fighting in rats was a function of both enclosed floor area and shock intensity. Manipulating sex of the subject, strain, previous familiarity with other subjects, and number of subjects present during shock did not alter the stereotyped pattern of fighting that was observed. Typically, when exposed to foot shock, paired rats assumed an upright posture, bared their teeth, and struck vigorously at each other with their forepaws. Optimal conditions for inducing fighting were defined as two rats confined in an experimental chamber exposed to a 2-mA footshock.

Azrin, Rubin, and Hutchinson (1968) identified a major problem found in most shock-elicited aggression studies, namely, the reliance upon subjective evaluation of movements and postures of the subject pairs that were considered to be aggressive. The ideal situation would be that of observing aggression in a single subject, thus eliminating the need for evaluation of specific movements and postures. Unfortunately, Ulrich and Azrin (1962) found that a lone rat typically does not aggress toward an inanimate object in response to footshock. Desiring to develop a technique whereby automatic recordings could be obtained for aggressive responses, Azrin et al. (1968) described a method by which individual rats could be made to bite an inanimate target object. In this procedure, restrained rats received unavoidable tailshocks of $5 \mathrm{~mA}$ intensity with a 200 -msec duration every $10 \mathrm{sec}$ for $20 \mathrm{~min}$. Results

This research was supported, in part, by a Tower Fund Research Grant from Austin Peay State University of the third author. Requests for reprints should be addressed to Stephen F. Davis, Department of Psychology, Austin Peay State University, Clarksville, Tennessee 37040 . of this investigation clearly revealed that biting attacks toward inanimate targets could be elicited by applying tailshock to restrained rat subjects. Consequently, the pain-aggression reaction in rats was more readily accessible to objective measurement and study.

Surprisingly, the effects of specific deprivation states on shock-induced aggression has received scanty attention in the literature. Cahoon, Crosby, Dunn, Herrin, Hill, and McGinnis (1971) determined that the effect of food deprivation on reflexive fighting was to increase, to a point, exhibited levels of aggression. Similarly, Devine (1971) and Hamby and Cahoon (1971) reported that frequency of shockinduced fighting was a function of level of water deprivation. The function seemed to be described best as curvilinear. In a related study, Bisbee and Cahoon (1973) found that lithium chloride induced nausea produced high levels of fighting in rat subjects at small dosage levels while larger doses served to inhibit display of shock-elicited aggression.

Within the context of deprivation and resultant drive enhancement, it would appear that some functional relationship might be obtained between deprivation of rapid eye movement (REM) sleep and reflexive fighting in rats. A phenomenon first described by Aserinsky and Kleitman (1953), REM sleep has frequently been described as the stage of sleep during which dreaming occurs (Dement \& Kleitman, 1957). As reported by Aserinsky and Kleitman (1953), rapid eye movements were typically accompanied by changes in respiration and heart rate, and changes in EEG pattern from the usual large, slow, regular wave pattern of deep sleep to a low-voltage, desynchronized one similar to that of an alert waking state.

Although relatively little is known regarding the behavioral effects of REM deprivation in animals (Stern, 1969), certain behaviors are sensitive to REM deprivation. For example, Pearlman (1971) reported that REM sleep deprivation impaired latent learning 
in rats. In a corollary publication, Pearlman (1973) also determined that REM sleep deprivation had a deleterious effect upon latent extinction in rat subjects. In a related series of studies, Stern (1971) found that acquisition of three tasks-passive avoidance, active avoidance, and appetitive alternation discrimination-was markedly impaired by 5 days of REM deprivation.

Other investigators have found that some behaviors may be enhanced as a function of REM deprivation. Hicks and Paulus (1973) ascertained that $0,24,48$, or $72 \mathrm{~h}$ of REM deprivation produced a significant inverse effect on latency of $T$-maze performance. These results were interpreted as supporting the contention that REM deprivation tends to increase generalized drive, an idea previously suggested by Dement, Henry, Cohen, and Ferguson (1967). Similarly, in a series of four experiments, Albert, Cicala, and Siegel (1970) found that both shuttle avoidance and runway avoidance were unaffected by 3,6 , or 9 days of REM deprivation. Increasing REM deprivation resulted in an enhancement of activity. These investigators also adopted a motivational-effects model. They posited that REM deprivation increased sensitivity of the REMdeprived subjects to environmental stimulation. Although not directly related to the area of shockelicited aggression, these studies (Albert et al., 1970; Hicks \& Paulus, 1973) are mentioned to introduce the motivational-effects model which will be discussed in relation to this paper.

A direction for REM studies to follow appears to be that of shock-elicited aggression. Stern (1969) reported that after 5 days of REM deprivation, using the water tank island method, rats subjected to REM deprivation manifested significantly lower aggression thresholds than either stress controls (those subjects partially immersed in cold water for 20 min per day over the course of aggression testing) or typical control subjects (those subjects maintained on large islands in the water tank apparatus). In a previous two-experiment series, Morden, Conner, Mitchell, Dement, and Levine (1968) deprived male LongEvans rats of REM sleep for 7 days. Shock intensity was also varied systematically across three equal groups of the subjects. In Experiment 1, all of the subjects were tested for aggression on alternate days for 7 days. Experiment 2 replicated Experiment 1, except that the subjects were tested after Day 7 only. Results of these manipulations indicated that the subjects deprived of REM sleep exhibited higher fighting frequencies than controls, particularly at low shock intensities. The fact that level of REM deprivation was a within-subjects variable introduced the possibility of confounding.

\section{EXPERIMENT 1}

The purpose of Experiment 1 was to systematically investigate the effects of level of REM deprivation on shock-elicited aggression in rats. Unlike the Morden et al. (1968) and Stern (1969) studies, REM deprivation was made a between-subjects factor so that each rat was tested only once. Thus, the possibility of confounding was eliminated. Unlike Morden et al. (1968), measures of shock-elicited aggression were obtained for each of the specified levels of REM deprivation as opposed to testing on alternate days of REM deprivation. In view of previous research concerned with the effects of REM deprivation on drive [i.e., REM deprivation is presumed to enhance generalized drive (Dement et al., 1967; Hicks \& Paulus, 1973)], it was specifically hypothesized that a linear function would be obtained between mild to moderate levels of REM deprivation and shockelicited aggression. In view of the previous research (Bisbee \& Cahoon, 1973; Cahoon, Crosby, Dunn, Herrin, Hill, \& McGinnis, 1971; Devine, 1971; Hamby \& Cahoon, 1971) relating shock-elicited aggression and deprivation, it was believed that a curvilinear relationship might be obtained with more severe deprivation levels.

\section{Method}

Subjects. The subjects were 36 male, albino rats purchased from the Holtzman Co., Madison, Wisconsin. The subjects were approximately 150 days old.

REM deprivation apparatus. The REM deprivation apparatus was similar to that described by Hicks and Paulus (1973). More specifically, during REM deprivation periods all subjects were maintained in 5-gallon metal pails, the tops of which were covered with $1.3-\mathrm{cm}$ hardware cloth. A water bottle and a metal food container were affixed to the hardware cloth such that each subject had free access to both food and water while confined to the pail. Inverted flower pots with bases measuring $7.4 \mathrm{~cm}$ and $11.2 \mathrm{~cm}$ in diameter served as islands for the subjects in the REM and control groups, respectively. Each pail was filled with water to within approximately $1.14 \mathrm{~cm}$ of the top of the inverted flower pot. Use of this technique precludes REM sleep, since animals typically lose muscle tonus at the onset of REM. Consequently, the subject either awakens or falls into the water. Amount of non-REM sleep, however, is not significantly altered since muscle tonus is maintained during non-REM periods (Jouvet, 1963). Plastic maintaining tanks with appropriately sized platforms were utilized to house the animals while water from the deprivation tanks was being changed. To reduce odors and the possibility of disease, the water in each tank was changed daily.

Shock-elicited aggression apparatus. A rat restraining device similar to that described by Azrin et al. (1968) served as the apparatus in shock-elicited aggression testing. This apparatus consisted of an opaque plastic tube, measuring $21.5 \mathrm{~cm}$ in length and $7.5 \mathrm{~cm}$ in diameter, mounted on a Plexiglas sheet. The Plexiglas sheet was, in turn, stabilized on a wooden platform to facilitate placement of the subject into the tube and to permit easy removal of fecal material and urine that accumulated in the tube during testing. A $1.5-\mathrm{cm}$ hole at the enclosed end of the tube 
allowed the subject's tail to be extended from the apparatus and secured to a wood restraining rod by means of adhesive tape. The other end of the tube was open. Two pieces of No. 14 copper wire, permanently attached to the rod $7 \mathrm{~cm}$ apart, served as tail electrodes. When the rod was in place, it served as both a restraining device to prohibit unauthorized escape from the apparatus and as an electrode carrier. A $1.5-\mathrm{mA}$ rectified current was used and was monitored by a Jackson (Model 665-J-2) milliammeter. The aggression target was a Lafayette Instrument $\mathrm{Co}$. omnidirectional lever (Model 80111). This lever was mounted on the wood platform, perpendicular to the open end of the restraining tube and parallel to the wood platform on which the tube was mounted. When the tube was in place on the platform, the lever extended across the open midportion of the end of the tube. The lever was $1.5 \mathrm{~cm}$ from the tube and required a movement of $1 \mathrm{~cm}$ to activate the microswitch. Closure of the microswitch, in turn, activated a Lafayette (Model 5707PS) impulse counter.

Procedure. At the beginning of the experiment, the subjects were randomly assigned to one of four equal groups: Group $\mathrm{C}$ (control, no REM deprivation), Group 24-R (24-h REM deprivation), Group 48-R (48-h REM deprivation), and Group 72-R (72-h REM deprivation). Subjects in Group $C$ were, in turn, randomly assigned to one of three subgroups $(24,48$, and 72 h) to provide appropriate time-in-tank controls.

On Day 1, subjects were placed on the inverted flower pots at 30 -min intervals in order to insure an individual testing period for each subject following the confinement-in-the-tank period. Subjects in Group $C$ were placed on the large $(11.2-\mathrm{cm})$ pots while subjects in Groups $24-R, 48-R$, and $72-R$ were placed on the small $(7.4-\mathrm{cm})$ pots. The order for placing subjects into the deprivation tanks, and hence the order for running subjects in the subsequent shock-elicited aggression task, was random.

Upon completion of the specified confinement period for each subject, the subject was removed from the deprivation tank and secured in the restraining tube. Prior to taping the restraining rod to the animal's tail, electrode paste was applied to the electrodes. The subject was positioned in the tube such that its nose was approximately $1 \mathrm{~cm}$ from the target rod. Each subject experienced a 5-min habituation period in the restraining tube prior to the administration of shock. A 15-min period of shock administration immediately followed habituation. During this time, each subject was exposed to a series of 300 -msec-duration $1.5-\mathrm{mA}$ shocks administered at 3-sec intervals. Thus, each subject experienced a total of 300 shocks. The total number of aggressive responses was recorded for each subject.

\section{Results}

The response data were transformed into $\log _{10}\left(X_{i}\right.$ $+1)$ scores prior to analysis of variance. Subsequent comparisons of significant effects were performed by the Newman-Keuls procedure. Group means are shown in Figure 1.

Prior to overall analysis, $\mathrm{F}$ max tests were performed on the data from Group $C$ in order to insure the propriety of pooling. The results of these analyses yielded nonsignificance, $F(2,2)=8.65, \mathrm{p}>.10$. Of course, it may be that confining Group $C$ subjects on the large-diameter pots had an effect on aggressiveness. A comparison ( $t$ test) of Group $C(\bar{X}=1.51)$ with a group of animals (unpublished data) confined in the normal home cage $(\overline{\mathrm{X}}=1.35$ ) was not significant, but did suggest a slight increase in aggressiveness.

Analysis of variance yielded significance for the Groups factor, $F(3,32)=3.61, p<.05$. Subsequent comparisons indicated that Groups $48-R$ and $72-R$

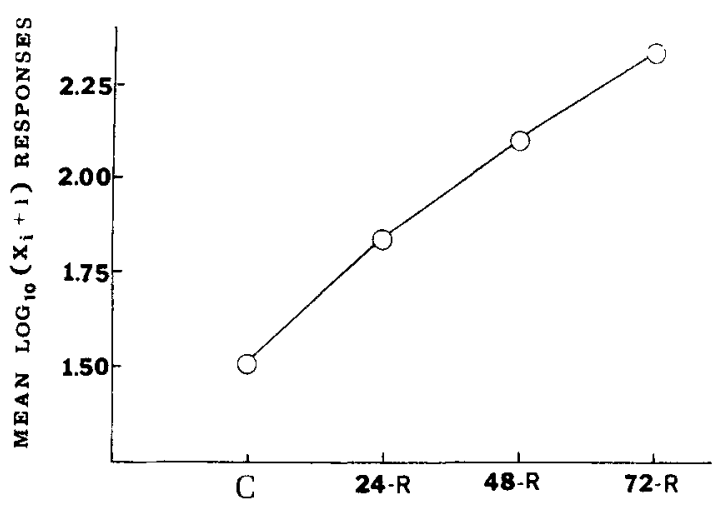

Figure 1. Group mean aggressive responses, Experiment 1.

were significantly $(p<.05)$ more aggressive than Group 24-R.

In view of past research on shock-elicited aggression, the shape of the obtained function is of interest. Tests for trend were performed. A significant linear trend, $F(1,32)=10.42, p<.01$, and a nonsignificant departure from linearity, $F(2,32)$ $=.15, \mathrm{p}>.25$, were found.

\section{Discussion}

The present study suggests that the amount of shock-induced aggression shown by rats is an increasing linear function of REM deprivation up to $72 \mathrm{~h}$ and is consistent with the data reported by Stern (1969) and Morden et al. (1968), who found that REM-deprived rats ( 5 and 7 days) were more aggressive than controls.

Although not statistically significant, a considerable amount of within-group variability was found in the present study. This observation is also consistent with that of other investigators working in the general area of shock-elicited aggression (Azrin, Hutchinson, \& Hake, 1963, 1966; Cahoon et al., 1971).

The results of the present study do not appear to be completely compatible with those of previous studies investigating the effects of other deprivation states on shock-induced aggression. For example, Cahoon et al. (1971) reported a curvilinear function between shock-induced fighting and level of food and water deprivation. Perhaps this discrepancy could be attributed to the fact that the uppermost level of REM deprivation employed in this study was not severe enough to produce the decrements observed under other deprivation states.

\section{EXPERIMENT 2}

Experiment 2 was prompted by the positive relationship between aggressive responding and length of REM deprivation shown in Experiment 1. Through the addition of longer REM-deprivation periods, one should be able to ascertain at what point this deprivation stage begins to have a detrimental effect 
upon aggression. More specifically, in Experiment 2 REM deprivation was extended to $120 \mathrm{~h}$. Additionally, a group receiving both 48-h REM deprivation and food deprivation was run. It was believed that the addition of this group would provide information on the effect of two simultaneous deprivation states on aggressive responding.

\section{Method}

Subjects. Forty male albino rats purchased from the Holtzman Co., Madison, Wisconsin, served as subjects. The subjects were approximately 150 days old.

Apparatus. The REM-deprivation apparatus and shock elicited aggression apparatus used in Experiment 1 were used in Experiment 2.

Procedure. Five equal groups were formed randomly at the beginning of the experiment. Groups 48-R, 72-R, 96-R, and 120-R received a corresponding amount of REM deprivation (in hours) prior to aggression testing. Subjects in Group $48+$ received both 48-h REM deprivation and food deprivation prior to aggression testing. Food deprivation consisted of restricting feeding to $12-14 \mathrm{~g}$ per day and was begun 2 days before the subjects were placed in the REM-deprivation tanks.

Both REM-deprivation and aggression-testing procedures were identical to those used in Experiment 1. The order for placing subjects in the REM-deprivation tanks, and hence the order for aggression testing, was determined randomly.

\section{Results and Discussion}

Mean aggressive responding for Experiment 2 is shown in Figure 2. Analysis of variance yielded a significant, $F(4,35)=2.76, \mathrm{p}<.05$, Groups effect. A subsequent Newman-Keuls test indicated that Group 96-R showed significantly $(p<.05)$ more aggressive responses than did all other groups, and that Group $48+$ was significantly $(p<.05)$ more aggressive than Groups $48-R$ and $72-R$. Trend analysis was performed on the data from Groups $48-R$ 72-R, 96-R, and 120-R (REM deprivation only). This analysis yielded a significant, $\mathrm{F}(1,32)=8.68, \mathrm{p}<.01$, quadratic trend, thus supporting the graphical impression that a curvilinear function best describes the relationship between REM deprivation and aggressive responding for these groups.

A possible problem is suggested by comparing the aggressiveness of Groups 48-R and 72-R between the two experiments. As can be seen from Figures 1 and 2, there appears to be more aggressiveness on the part of both Groups 48-R and Group 72-R in Experiment 2. Thus, the question of reliability of results might be raised, although such differences are not totally unexpected. Previous research (e.g., Azrin, Hutchison, \& Hake, 1963; Cahoon et al., 1971) in this area has been characterized by a large amount of within-group variability. It is not unlikely that this factor contributed to the observed differences. Also, the use of different litters (even though obtained from the same supplier) may have contributed to the differences. On the other hand, it should be noted that the pattern of results is consistent between the two experiments (i.e., Group 48-R was less aggressive

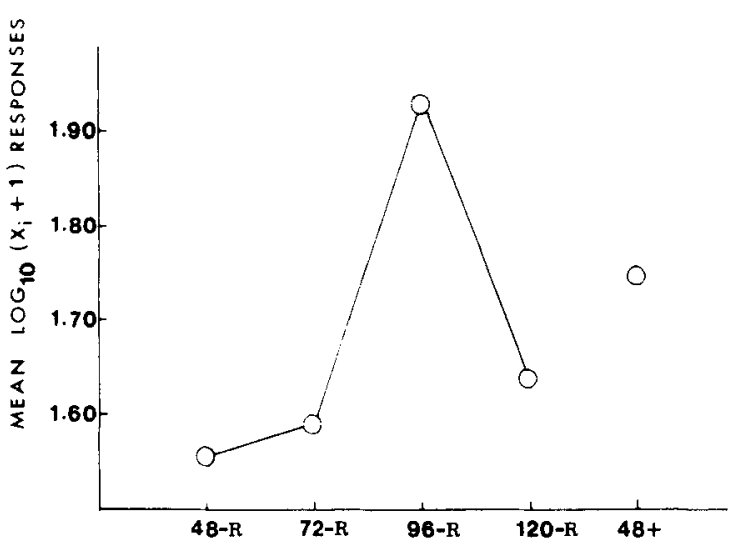

Figure 2. Group mean aggressive responses, Experiment 2.

than Group 72-R in both instances). Along these lines, the significant linear trend observed in Experiment 1 is paralleled by a similar $(p<.05)$ linear trend among Groups 48-R, 72-R, and 96- $R$ in Experiment 2. Thus, both studies support the notion that increases in REM sleep deprivation, up to a point (somewhere between 96 and $120 \mathrm{~h}$, judging from the present data), result in increases in aggressiveness.

\section{GENERAL DISCUSSION}

Considering the results of both studies, several interesting patterns emerge. First, up to a point (96 $\mathrm{h}$ in Experiment 2), increases in REM deprivation result in corresponding increases in the number of aggressive responses. These results are in general agreement with previous REM studies (Morden et al., 1968; Stern, 1969). Second, the decreased number of aggressive responses shown by Group 120-R (Experiment 2) indicates that longer REM-deprivation periods may serve to decrease aggressiveness. This result is in agreement with previous deprivation studies (Bisbee \& Cahoon, 1973; Cahoon et al., 1971; Devine, 1971; Hamby \& Cahoon, 1971) that have reported a curvilinear relation between deprivation level and shock-elicited aggression.

Concerning a possible mechanism underlying these results, it would appear that a drive-enhancement or motivational-effects model is a likely candidate. As already noted, this explanation has been used by previous studies attempting to relate deprivation level and aggression. Hence its use here would not be unwarranted. The increased aggressiveness shown by Group $48+$ (Experiment 2) also appears supportive of this interpretation. The combination of food and REM deprivation for this group presumably resulted in increased drive. This is reflected by the significant increase in aggressive responding shown by this group relative to Group 48-R (Experiment 2 ) which received only REM deprivation.

However, one problem still remains if one is to accept the drive-energization or motivational-effects 
model. Namely, what is causing the decrease in aggression shown by Group 120-R (Experiment 2). Such decreases in performance frequently observed with relatively severe increases in traditional deprivation states, such as food and water, may be explained in terms such as an "energy deficit" (see Bolles, 1967). Such an energy deficit would be expected to increase in some fashion with time of deprivation, and would, at some point, override the energizing effects. On the other hand, terms like "energy deficit" or "tissue depletion" would appear to be tenuous, at best, when applied to REM deprivation. Hence the problem and the need for a fuller understanding of the mechanisms underlying REM deprivation.

Although speculative, it may well be that the decrease in aggressiveness shown by Group 120-R in Experiment 2 is due to a lack of brain catecholamine synthesis. In support of this, Stern and Morgane (1974) have recently proposed that brain catecholamines are synthesized during REM sleep. Applying this notion to the present data, it would appear that REM deprivation has an energizing effect up to the point at which decreased catecholamine levels impair neural processing. Although the exact time course for catecholamine depletion has yet to be fully delineated, it is suggestive to note that behavioral data reported by Stern and Morgane (1974) are consistent with this interpretation of these data. They reported that injections of imipramine (a drug that increases catecholamine activity) resulted in a significant increase in active avoidance learning on the part of animals deprived of REM sleep for 5 days $(120 \mathrm{~h})$. Imipramine injections failed to enhance such acquisition in normal and stress control animals.

\section{REFERENCES}

Albert, I., Cicala, G. A., \& Siegel, J. The behavioral effects of REM sleep deprivation in rats. Psychophysiology, 1970, 6, $550-560$.

Aserinsky, E.. \& Kzeitman, N. Regularly occurring periods of eye motility and concomitant phenomena during sleep. Science, 1953, 118, 273-274.

Azrin, N. H., Hutchinson, R. R., \& Hake, D. F. Pain-induced fighting in the squirrel monkey. Joumal of the Experimental Analysis of Behavior, 1963, 6. 620.

Azrin, N. H., Hutchinson, R. R., \& Hake, D. F. Extinction- induced aggression. Joumal of the Experimental Analysis of Behavior. 1966, 9.191-204.

Azrin. N. H., Rubin, H. B., \& Hutchinson, R. R. Biting attack by rats in response to aversive shock. Journal of the Experimental Analysis of Behavior, 1968, 11, 633-639.

BisbeE. D. S. P., \& Cahoon, D. D. The effects of induced nausea upon shock-elicited aggression. Bulletin of the Psychonomic Society, 1973, 1, 19-21.

Bolles, R. C. Theory of motivation. New York: Harper and Row, 1967.

Cahoon, D. D.. Crosby, R. M., Dunn, S., Herrin, M. S., Hill, C. C., \& McGinnis, M. The effect of food deprivation on shockelicited aggression in rats. Psychonomic Science, 1971, 22, 43-44.

Dement, W., Henry, P., Cohen, H., \& Ferguson, J. Studies on the effect of REM deprivation in humans and animals. Publications of the Association for Research on Nervous and Mental Diseases, 1967, 45, 456-468.

Dement, W. C., \& Kleitman, N. Cyclic variations in EEG during sleep and their relations to eye movements, body motility, and dreaming. Electroencephalography and Clinical Neurophysiology, 1957. 9. 673-690.

DEvine, M. D. A parametric investigation into the effects of water deprivation on shock elicited aggression. Unpublished master's thesis. Auburn University, 1971.

Hamby, W. G., \& CAHOON, D. D. The effect of water deprivation upon shock elicited aggression in the white rat. Psychonomic Science, 1971, 25, 166-167.

Hicks, R. A. \& P Pulus. M. J. Effects of rapid eye movement sleep deprivation on the performance of rats in a T-maze. Psychological Record, 1973, 23, 89-92.

Jouvet, M. An automatic recorder of the rhombencephalic phases of sleep in the cat: The oneirograph. EEG in Clinical Neurophysiology, 1963, 15, 141.

Morden, B., Conner, R.. Mitchell, G., Dement, W., \& LeVINE, S. Effects of rapid eye movement (REM) sleep deprivation on shock-induced fighting. Physiology and Behavior, 1968 , 3, 425-432.

O'Kelly, L. I., \& Steckle, L. C. A note on long enduring emotional responses in the rat. The Journal of Psychology, 1939, 8. 125-131.

Pearlman, C. REM sleep deprivation impairs latent extinction in rats. Physiology and Behavior, 1973, 11, 233-237.

Pearlman, C. A., JR. Latent learning impaired by REM sleep deprivation. Psychonomic Science, 1971, 25, 135-136.

STERN, W. C. Behavioral and biochemical aspects of rapid eye movement deprivation in the rat. Unpublished doctoral dissertation, Indiana University, 1969.

Stern, W. C. Acquisition impairments following rapid eye movement sleep deprivation in rats. Physiology and Behavior, 1971, 7. 345-352.

Stern, W. C., \& Morgane, P. J. Theoretical view of REM sleep function: Maintenance of catecholamine systems in the central nervous system. Behavioral Biology, 1974, 11, 1-32.

Ulrich, R. E., \& AzRIN, N. H. Reflexive fighting in response to aversive stimulation. Journal of the Experimental Analysis of Behavior, 1962, 5. 511-520.

(Received for publication August 20.1976; revision accepted December 9.1976 .) 